International Journal of Pure and Applied Mathematics

Volume 87 No. 1 2013, 9-18

ISSN: 1311-8080 (printed version); ISSN: 1314-3395 (on-line version)

url: http://www.ijpam.eu

doi: http://dx.doi.org/10.12732/ijpam.v87i1.2

ijpam.eu

\title{
SYMMETRIC BI-DERIVATIONS ON TM-ALGEBRAS
}

\author{
T. Ganeshkumar ${ }^{1}$, M. Chandramouleeswaran ${ }^{2} \S$ \\ ${ }^{1}$ Department of Mathematics \\ M.S.S. Wakf Board College \\ Madurai, 625020, INDIA \\ ${ }^{2}$ Department of Mathematics \\ SBK College \\ Arupukottai, 626101, INDIA
}

\begin{abstract}
Recently an algebra based on propositional calculi was introdued by Tamilarasi and Mekalai in the year 2010 known as $T M$-algebras, see [6]. In our paper [1] we introduced the notion of derivation on TM-algebras. In this paper, we introduce the notion of symmetric bi-derivation on TM-algebras and study some of its properties.
\end{abstract}

AMS Subject Classification: 03G25, 06F35

Key Words: BCK/BCI algebras, TM-algebras, derivations, symmetric biderivations

\section{Introduction}

It is well known that BCK and BCI-algebras are two classes of algebras of logic. They were introduced by Imai and Iseki [3] and have been extensively investigated by many researchers. Recently another algebra based on propositional calculi was introdued by Tamilarasi and Mekalai [6] in the year 2010 known as $T M$-algebras.

Motivated by the notion of derivations on rings and near-rings Jun and Xin [4] studied the notion of derivation on BCI-algebras. In our paper [1],

Received: $\quad$ March 21, 2013

(C) 2013 Academic Publications, Ltd.

$\S$ Correspondence author url: www.acadpubl.eu 
we introduced the notion of derivation on $T M$-algebras. In [5], the authors have discussed the notion of symmetric bi-derivation on BCI-algebras. This motivated us to introduce the notion of symmetric bi-derivation on TM-algebras in this paper. We study the properties of symmetric bi-derivations on TMalgebras and prove that the set of all symmetric bi-derivations on a TM-algebra forms a semigroup under a suitably defined binary composition.

\section{Preliminaries}

In this section, we recall some basic definitions and results that are needed for our work.

Definition 2.1. A $T M$-algebra $(X, *, 0)$ is a non-empty set $X$ with a constant 0 and a binary operation $*$ satisfying the following axioms:

1. $x * 0=x$

2. $(x * y) *(x * z)=z * y \forall x, y, z \in X$.

Definition 2.2. A $T M$-algebra $X$ is said to be associative if $(x * y) * z=$ $x *(y * z)$ for all $x, y, z \in X$.

Definition 2.3. For any $T M$-algebra $(X, *, 0)$. We define the set $G(X)=$ $\{x \in X \mid 0 * x=x\}$.

Remark 2.4. In a $T M$-algebra $X$, by definition, $x \wedge y=y *(y * x)$. However, in a TM-algebra, $x=y *(y * x)$. Hence, in a TM-algebra, we have $x \wedge y=x \quad \forall x, y \in X$.

Definition 2.5. [2] Let $X$ be a $T M$-algebra. If we define an operation + , called addition, as $x+y=x *(0 * y)$ for all $x, y \in X$, then $(X,+)$ is an abelian group with identity 0 and the additive inverse $-x=0 * x \forall x \in X$.

Remark 2.6. If we have a $T M$-algebra $(X, *, 0)$ it follows from the above definition that $(X,+)$ is an abelian group with $-y=0 * y \forall y \in X$. Then we have $x-y=x * y \forall x, y \in X$. On the other hand if we choose an abelian group $(X,+)$ with an identity 0 and define $x * y=x-y$, we get a $T M$-algebra $(X, *, 0)$ where $x+y=x *(0 * y) \quad \forall x, y \in X$.

Definition 2.7. Let $(X, *, 0)$ be a $T M$-algebra. A self map $d: X \rightarrow X$ is said to be a $(l, r)$-derivation on $X$, if $d(x * y)=(d(x) * y) \wedge(x * d(y))$. $d$ is said to be a $(r, l)$-derivation on $X$, if $d(x * y)=(x * d(y)) \wedge(d(x) * y)$. It is said to be a derivation on $X$ if $d$ is both a $(l, r)$-derivation and a $(r, l)$-derivation on $X$. 


\section{Symmetric BI-Derivations}

We start this section with the definition of Cartesian product of $T M$-algebras.

Definition 3.1. Let $X, Y$ be $T M$-algebras. An operation $*$ on the Cartesian product $X \times Y$ of $X, Y$ is defined as follws.

1. $\left(x_{1}, y_{1}\right) *\left(x_{2}, y_{2}\right)=\left(x_{1} * x_{2}, y_{1} * y_{2}\right)$.

2. $(0,0)=0$,

Lemma 3.2. Cartesian product of two $T M$-algebras is again a $T M$-algebra.

Proof. Let $X$ and $Y$ be two $T M$-algebras. Consider the cartesian product $X \times Y$.

$$
\begin{aligned}
& (x, y) *(0,0)=(x * 0, y * 0)=(x, y) \\
\left(\left(x_{1}, y_{1}\right) *\left(x_{2}, y_{2}\right)\right) *\left(\left(x_{1}, y_{1}\right) *\left(x_{3}, y_{3}\right)\right)=\left(x_{1} * x_{2}, y_{1} * y_{2}\right) *\left(\left(x_{1} * x_{3}, y_{1} * y_{3}\right)\right) & =\left(\left(x_{1} * x_{2}\right) *\left(x_{1} * x_{3}\right),\left(y_{1} * y_{2}\right) *\left(y_{1} * y_{3}\right)\right) \\
= & \left(\left(x_{3} * x_{2}\right),\left(y_{3} * y_{2}\right)\right) \\
= & \left(x_{3}, y_{3}\right) *\left(x_{2}, y_{2}\right)
\end{aligned}
$$

Therefore $(X \times Y, *, 0)$ is a $T M$-algebra.

Definition 3.3. Let $X$ be a $T M$-algebra. A mapping $D: X \times X \rightarrow X$ is a symmetric map if $D(x, y)=D(y, x)$ holds for all pairs of elements $x, y \in X$.

Example 3.4. Let $(X, *, 0)$ be a $T M$-algebra with the Cayley table.

\begin{tabular}{|l|l|l|l|l|}
\hline$*$ & 0 & 1 & 2 & 3 \\
\hline 0 & 0 & 1 & 2 & 3 \\
\hline 1 & 1 & 0 & 3 & 2 \\
\hline 2 & 2 & 3 & 0 & 1 \\
\hline 3 & 3 & 2 & 1 & 0 \\
\hline
\end{tabular}

The map $D: X \times X \rightarrow X$ defined by $D(x, y)=x *(0 * y)$ is a symmetric map.

Definition 3.5. Let $X$ be a $T M$-algebra and $D: X \times X \rightarrow X$ be a symmetric mapping. A mapping $d: X \rightarrow X$ defined by $d(x)=D(x, x)$ is called trace of $D$. 
Example 3.6. Let $(X, *, 0)$ be a $T M$-algebra with the Cayley table.

\begin{tabular}{|l|l|l|l|l|}
\hline$*$ & 0 & 1 & 2 & 3 \\
\hline 0 & 0 & 2 & 1 & 3 \\
\hline 1 & 1 & 0 & 3 & 2 \\
\hline 2 & 2 & 3 & 0 & 1 \\
\hline 3 & 3 & 1 & 2 & 0 \\
\hline
\end{tabular}

The map $D: X \times X \rightarrow X$ defined by $D(x, y)=x *(0 * y)=x+y$ is a symmetric map.

Since $x=0, D(0,0)=0+0=0 . x=1, D(1,1)=1+1=3 . x=2$, $D(2,2)=2+2=3 . x=3, D(3,3)=3+3=0$.

Thus the mapping $d: X \rightarrow X$ given by $d(x)=D(x, x)= \begin{cases}0 & \text { if } x=0,3 \\ 3 & \text { if } x=1,2\end{cases}$ is the trace of the symmetric mapping $D$.

Definition 3.7. Let $X$ be a $T M$-algebra and $D: X \times X \rightarrow X$ be a symmetric mapping. If $D$ satisfies the identity, $D(x * y, z)=(D(x, z) * y) \wedge(x *$ $D(y, z))$ for all $x, y, z \in X$, then $D$ is called left-right symmetric bi-derivation. ( $(l, r)$ symmetric bi-derivation)

If $D$ satisfied the identity, $D(x * y, z)=(x * D(y, z)) \wedge(D(x, z) * y)$ for all $x, y, z \in X$, then $D$ is called right-left symmetric bi-derivation. $((r, l)$-symmetric bi-derivation)

If $D$ is both an $(l, r)$ symmetric bi-derivation and an $(r, l)$ symmetric biderivation then $D$ is called a symmetric bi-derivation.

Example 3.8. Consider in example 3.6. Define a mapping $D: X \times X \rightarrow X$ by $D(x, y)=x *(0 * y)$ for all $x, y \in X$. Then $D$ is a $(l, r)$-symmetric Biderivation.

Example 3.9. Consider in example 3.4. Define $D(x, y)=x *(0 * y)$ for all $x, y \in X$ is a symmetric map. Then $D$ is a symmetric Bi-derivation.

Example 3.10. Consider the $T M$-algebra with the Cayley-Table as in exapmle 3.4. Define the symmetric map $D: X \times X \rightarrow X$ such that

$D(x, x)=3$, if $x=0,1,2,3$.

$D(0,3)=D(3,0)=D(1,2)=D(2,1)=0$.

$D(0,2)=D(2,0)=D(1,3)=D(3,1)=1$.

$D(0,1)=D(1,0)=D(2,3)=D(3,2)=2$.

Then $D$ is a symmetric Bi-derivation.

Proposition 3.11. Let $X$ be a $T M$-algebra. Define a symmetric map $D: X \times X \rightarrow X$ by $D(x, y)=x+y$ for all $x, y \in X$. Then $D$ is a $(l, r)$-symmetric 
Bi-derivation.

Proof.

$$
\begin{aligned}
D(x * y, z) & =(x * y)+z \quad \text { for all } x, y, z \in X \\
& =(x * y) *(0 * z) \\
& =(x *(0 * z)) * y \quad(\because(x * y) * z=(x * z) * y) \\
& =(x+z) * y \\
& =(x *(y+z)) *((x *(y+z)) *((x+z) * y))(\because y *(y * x)=x) \\
& =((x+z) * y) \wedge(x *(y+z)) \\
& =(D(x, z) * y) \wedge(x * D(y, z))
\end{aligned}
$$

This proves that $D$ is a $(l, r)$-symmetric Bi-derivation.

Theorem 3.12. Let $X$ be an associative $T M$-algebra. Then the symmetric map $D: X \times X \rightarrow X$ defined by $D(x, y)=x+y$ for all $x, y \in X$ is a symmetric bi-derivation.

Proof. By the above proposition, $D$ is a $(l, r)$-symmetric bi-derivation.

$$
\begin{aligned}
D(x * y, z) & =(x * y)+z \\
& =(x * y) *(0 * z) \\
& =(x *(0 * z)) * y \\
& =((x * 0) * z) * y \quad(\because X \text { is associative }) \\
& =(x * z) * y=(x * y) * z \quad \cdots \cdots(1) \\
(x * D(y, z)) \wedge(D(x, z) * y) & =x * D(y, z) \quad(\because x \wedge y=y *(y * x)=x) \\
& =x *(y+z) \\
& =x *(y *(0 * z)) \\
& =x *((y * 0) * z) \quad(\because X \text { is associative }) \\
& =x *(y * z) \\
& =(x * y) * z \quad \cdots \cdots(2) \quad(\because X \text { is associative })
\end{aligned}
$$

From (1) and (2), $D(x * y, z)=(x * D(y, z)) \wedge(D(x, z) * y)$ for all $x, y, z \in X$.

This proves that $D$ is $(r, l)$-symmetric bi-derivation and hence a symmetric bi-derivation.

Proposition 3.13. Let $X$ be a $T M$-algebra and $D: X \times X \rightarrow X$ be a symmetric map. Then 
1. If $D$ is a $(l, r)$-symmetric bi-derivation then $D(x, y)=D(x, y) \wedge x$ for all $x, y \in X$.

2. If $D$ is a $(r, l)$-symmetric bi-derivation then $D(x, y)=x \wedge D(x, y)$ for all $x, y \in X$ if and only if $D(0, y)=0$ for all $y \in X$.

Proof.

1. Let $D$ be a $(l, r)$-symmetric bi-derivation.

$$
\begin{aligned}
D(x, y)= & D(x * 0, y) \quad \text { for all } x, y \text { in } X \\
= & (D(x, y) * 0) \wedge(x * D(0, y)) \\
= & D(x, y) \wedge(x * D(0, y)) \\
= & (x * D(0, y)) *((x * D(0, y)) * D(x, y)) \\
= & (x * D(0, y)) *((x * D(x, y)) * D(0, y)) \\
& (\because(x * y) * z=(x * z) * y) \\
= & x *(x * D(x, y)) \quad(\because(x * z) *(y * z)=x * y) \\
= & D(x, y) \wedge x
\end{aligned}
$$

2. Let $D$ be a $(r, l)$-symmetric bi-derivation and $D(0, y)=0$ for all $y \in X$.

$$
\begin{aligned}
D(x, y) & =D(x * 0, y) \\
& =(x * D(0, y)) \wedge(D(x, y) * 0) \\
& =(x * 0) \wedge D(x, y) \\
& =x \wedge D(x, y) .
\end{aligned}
$$

Conversely, if $D(x, y)=x \wedge D(x, y)$ for all $x, y \in X$. Then

$$
D(0, y)=0 \wedge D(0, y)=D(0, y) *(D(0, y) * 0)=D(0, y) * D(0, y)=0
$$

Proposition 3.14. Let $X$ be a $T M$-algebra and $D: X \times X \rightarrow X$ be a $(l, r)$-symmetric bi-derivation. Then

1. $D(a, y)=D(0, y) *(0 * a)=D(0, y)+a$ for all $a, y \in X$.

2. $D(a+b, y)=D(a, y)+D(b, y)-D(0, y)$ for all $a, b, y \in X$.

3. $D(a, y)=a$ for all $a, y \in X$ if and only if $D(0, y)=0$.

Proof. 
1. Let $a=0 *(0 * a)$.

$$
\begin{aligned}
D(a, y) & =D(0 *(0 * a), y) \\
& =(D(0, y) *(0 * a)) \wedge(0 * D(0 * a, y)) \\
& =D(0, y) *(0 * a) \quad(\because x \wedge y=x) \\
& =D(0, y)+a
\end{aligned}
$$

2. $\operatorname{By}(1)$

$$
\begin{aligned}
D(a+b, y) & =D(0, y)+a+b \\
& =D(0, y)+a+D(0, y)+b-D(0, y) \\
& =D(a, y)+D(b, y)-D(0, y)
\end{aligned}
$$

3. $D(a, y)=a$ for all $a, y \in X$.

Put $a=0, D(0, y)=0 \quad \forall y \in X$.

Conversely if $D(0, y)=0$, then $D(a, y)=D(0, y)+a=0+a=a$.

Proposition 3.15. Let $X$ be a $T M$-algebra and $D: X \times X \rightarrow X$ be a $(r, l)$-symmetric bi-derivation. Then

1. $D(a, y) \in G(X)$ for all $a \in G(X)$.

2. $D(a, y)=a * D(0, y)=a+D(0, y)$ for all $a, y \in X$.

3. $D(a+b, y)=D(a, y)+D(b, y)-D(0, y)$ for all $a, b, y \in X$.

4. $D(a, y)=a$ for all $a, y \in X$ if and only if $D(0, y)=0$.

Proof.

1. $0 * a=a \quad(\because a \in G(X))$.

$$
\begin{aligned}
D(a, y) & =D(0 * a, y) \quad \text { for all } a, y \in X \\
& =(0 * D(a, y)) \wedge(D(0, y) * a) \\
& =(D(0, y) * a) *((D(0, y) * a) *(0 * D(a, y))) \\
& =0 * D(a, y) \quad(\because y *(y * x)=x)
\end{aligned}
$$

This shows that $D(a, y) \in G(X)$. 
2.

$$
\begin{aligned}
D(a, y) & =D(a * 0, y) \quad \text { for all } a, y \in X \\
& =(a * D(0, y)) \wedge(D(a, y) * 0) \\
& =(a * D(0, y)) \wedge D(a, y) \\
& =D(a, y) *(D(a, y) *(a * D(0, y))) \\
& =a * D(0, y)
\end{aligned}
$$

$$
\begin{aligned}
\operatorname{Again} D(a, y) & =a * D(0, y) \\
& =a * D(0 * 0, y) \\
& =a *((0 * D(0, y)) \wedge(D(0, y) * 0)) \\
& =a *(0 * D(0, y)) \\
& =a+D(0, y)
\end{aligned}
$$

3. $D(a+b, y)=a+b+D(0, y)=a+D(0, y)+b+D(0, y)-D(0, y)$

$$
=D(a, y)+D(b, y)-D(0, y) \text {. }
$$

4. If $D(0, y)=0$, then $D(a, y)=D(a * 0, y)=a * D(0, y)=a * 0=a$.

(2)) Conversely if $D(a, y)=a \forall a \in X, D(0, y)=0$.

\section{Semigroup of Symmetric Bi-Derivations}

Definition 4.1. Let $\mathscr{D}_{L}$ denote the set of all $(l, r)$-symmetric bi-derivation on $X$. Define the binary operation $\wedge$ on $\mathscr{D}_{L}$ as follows: For $D_{1}, D_{2} \in \mathscr{D}_{L}$ define $\left(D_{1} \wedge D_{2}\right)(x, y)=D_{1}(x, y) \wedge D_{2}(x, y)$ for all $x, y \in X$.

Proposition 4.2. Let $D_{1}$ and $D_{2}$ are $(l, r)$-symmetric bi-derivation on $X$, then $\left(D_{1} \wedge D_{2}\right)$ is also a $(l, r)$-symmetric bi-derivation.

Proof. We will prove the following implication

$$
\begin{aligned}
\left(D_{1} \wedge D_{2}\right)(x * y, z) & =\left(\left(D_{1} \wedge D_{2}\right)(x . z) * y\right) \wedge\left(x *\left(\left(D_{1} \wedge D_{2}\right)(y, z)\right)\right) \\
\left(D_{1} \wedge D_{2}\right)(x * y, z) & =D_{1}(x * y, z) \wedge D_{2}(x * y, z) \\
& =D_{2}(x * y, z) *\left(D_{2}(x * y, z) * D_{1}(x * y, z)\right) \\
& =D_{1}(x * y, z)
\end{aligned}
$$




$$
\begin{aligned}
= & \left(D_{1}(x, z) * y\right) \wedge\left(x * D_{1}(y, z)\right) \\
= & \left(x * D_{1}(y, z)\right) *\left(\left(x * D_{1}(y, z)\right) *\left(D_{1}(x, z) * y\right)\right) \\
= & D_{1}(x, z) * y \quad \cdots \cdots(1) \\
\left(\left(D_{1} \wedge D_{2}\right)(x, z) * y\right) \wedge & \left(x *\left(D_{1} \wedge D_{2}\right)(y, z)\right)=\left(x *\left(D_{1} \wedge D_{2}\right)(y, z)\right) * \\
& \left(\left(x *\left(D_{1} \wedge D_{2}\right)(y, z)\right) *\left(\left(D_{1} \wedge D_{2}\right)(x, z) * y\right)\right) \\
= & \left(D_{1} \wedge D_{2}\right)(x, z) * y \\
= & \left(D_{1}(x, z) \wedge D_{2}(x, z)\right) * y \\
= & \left(D_{2}(x, z) *\left(D_{2}(x, z) * D_{1}(x, z)\right)\right) * y \\
= & D_{1}(x, z) * y \quad \cdots \cdots(2)
\end{aligned}
$$

Combining (1) and (2), we get $\left(D_{1} \wedge D_{2}\right)$ is a $(l, r)$-symmetric bi-derivation.

Proposition 4.3. The binary composition $\wedge$ defined on $\mathscr{D}_{L}$ is associative.

Proof. Let $X$ be a $T M$-algebra. Let $D_{1}, D_{2}, D_{3}$ are $(l, r)$-symmetric biderivation.

$$
\begin{aligned}
\left.\left(\left(D_{1} \wedge D_{2}\right) \wedge D_{3}\right)(x * y, z)=\left(\left(D_{1} \wedge D_{2}\right)(x * y, z)\right) \wedge D_{3}(x * y, z)\right) & \\
= & \left(D_{1}(x, z) * y\right) \wedge\left(D_{3}(x * y, z)\right) \quad(\text { By proposition4.2(1)) } \\
= & D_{3}(x * y, z) *\left(D_{3}(x * y, z) * D_{1}(x, z) * y\right) \\
= & D_{1}(x, z) * y \\
\left(D_{1} \wedge(\right. & \left.\left.D_{2} \wedge D_{3}\right)\right)(x * y, z)=\left(D_{1}(x * y, z)\right) \wedge\left(\left(D_{2} \wedge D_{3}\right)(x * y, z)\right) \\
= & \left(D_{1}(x * y, z)\right) \wedge\left(D_{2}(x, z) * y\right) \quad(\text { By proposition } 4.2(1)) \\
= & \left(D_{2}(x, z) * y\right) *\left(\left(D_{2}(x, z) * y\right) *\left(D_{1}(x * y, z)\right)\right) \\
= & D_{1}(x * y, z) \\
= & \left(D_{1}(x, z) * y\right) \wedge\left(x * D_{1}(y, z)\right) \\
= & \left(x * D_{1}(y, z)\right) *\left(\left(x * D_{1}(y, z)\right) * D_{1}(x, z) * y\right) \\
= & D_{1}(x, z) * y .
\end{aligned}
$$

Combining (1) and (2) we get, $\left(D_{1} \wedge D_{2}\right) \wedge D_{3}=D_{1}\left(D_{2} \wedge D_{3}\right)$.

This proves that, $\wedge$ is associative.

Combining the above two propositions, we get the following theorem.

Theorem 4.4. $\mathscr{D}_{L}$ is a semigroup under the binary composition $\wedge$ defined by $\left(D_{1} \wedge D_{2}\right)(x, y)=D_{1}(x, y) \wedge D_{2}(x, y)$ for all $x, y \in X$ and $D_{1}, D_{2} \in \mathscr{D}_{L}$. 
Analogously we can prove that,

Theorem 4.5. $\mathscr{D}_{R}$ is a semigroup under the binary operation $\wedge$ defined by $\left(D_{1} \wedge D_{2}\right)(x, y)=D_{1}(x, y) \wedge D_{2}(x, y)$ for all $x, y \in X$ and $D_{1}, D_{2} \in \mathscr{D}_{R}$ where $\mathscr{D}_{R}$ is the set of all $(r, l)$-symmetric bi-derivation.

Combining the above two theorem we get the following theorem.

Theorem 4.6. If $\mathscr{D}$ denotes the set of all symmetric bi-derivation on $X$, it is a semi-group under the binary operation $\wedge$ defined by $\left(D_{1} \wedge D_{2}\right)(x, y)=$ $D_{1}(x, y) \wedge D_{2}(x, y)$ for all $x, y \in X$ and $D_{1}, D_{2} \in \mathscr{D}$.

\section{References}

[1] T. Ganeshkumar, M. Chandramouleeswaran, Derivations On TM-algerbas, International Journal of Mathematical Archive, 3, No. 11 (2012), 3967-3974.

[2] T. Ganeshkumar, M. Chandramouleeswaran, Generalized derivation on TM-algebras, International Journal of Algebra, Accepted.

[3] Y. Imai, K. Iseki, On axiom systems of propositional calculi, Proc. Japan Acad. Ser A: Math. Sci., 42 (1966), 19-22.

[4] Y.B. Jun, X.L. Xin, On derivations of BCI-algebras, Inform. Sci., 159 (2004), 167-176.

[5] Sabahattin Ilbira, Alev Firat, Y.B. Jun, On symmetric bi-derivations of BCI-algebras, Applied Mathematical Sciences, 5, No. 60 (2011), 2957-2966.

[6] A. Tamilarasi, K. Megalai, TM-algebra an introduction, CASCT (2010). 